\section{Pollenflugkalender für die Praxis}

Die Stiftung Deutscher Polleninformationsdienst hat 2008 erstmals nach 15 Jahren ihren Pollenflugkalender aktualisiert. Die Neuauflage war nötig geworden, da sich durch den Klimawandel besonders in den Herbst- und Wintermonaten Veränderungen im Pollenflug ergeben hatten. Ganz neu aufgenommen in den Kalender wurde außerdem das Traubenkraut (Ambrosia artemisiifolia).

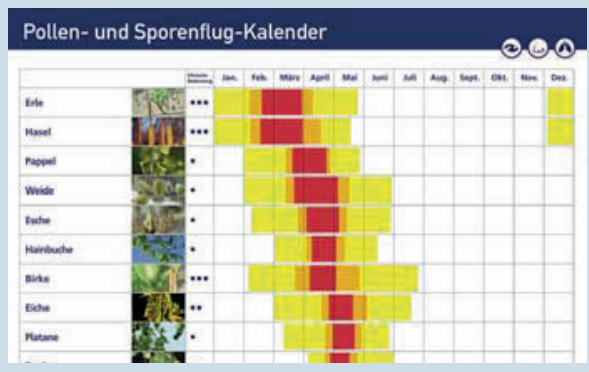

Das Unternehmen Bencard Allergie bietet im Rahmen seines Praxisservices den neuen Pollenflugkalender gleich in zwei Ausführungen an, zum einen im praktischen Scheckkartenformat für Patienten, zum anderen im Format DIN A2 zum Aufhängen in der Praxis. Beide Varianten können Allergologen kostenlos online im Servicebereich der Firmen-Homepage www.bencard-allergie.de bestellen.

Nach Informationen von Bencard, München

\section{Erweiterte Zulassung}

Das Antihistaminikum Aerius ${ }^{\circledR}$ hat in diesem Frühjahr von der europäischen Arzneimittelbehörde EMEA eine Indikationserweiterung erhalten. Das Präparat mit dem Wirkstoff Desloratadin kann jetzt bei allen Urtikariatypen eingesetzt werden, die bisherige Einschränkung auf die chronische idiopathische Form fällt weg. Die Zulassungserweiterung stimmt mit den aktuellen Leitlinien der internationalen Fachgesellschaften EAACI und $G A L^{2} E N$ überein. Danach gelten $\mathrm{H}_{1}$-Antihistaminika als First-Line-Therapie der Urtikaria. Moderne Wirkstoffe wie Desloratadin bewirken sowohl eine Kontrolle der durch Histamin induzierten Hautreaktionen der Frühphase als auch der inflammatorischen Symptome der Spätphase. Aerius ${ }^{\circledR}$ ist zudem nicht sedierend und es sind keine Wechselwirkungen mit Medikamenten oder Nahrungsmitteln bekannt.

Nach Informationen von essex pharma, München

\title{
Gräsertablette hat weiter die Nase vorn
}

$S^{c i n}$ it 2000 lässt die Fachzeitschrift PharmaBarometer jedes Jahr in einer Meinungsumfrage in verschiedenen Facharztgruppen das Image von Pharmaunternehmen und deren Produkten ermitteln. Die Auszeichnung für das beliebteste Unternehmen, die Goldene Tablette, und der Preis für das innovativste Produkt gelten als eine Art „Oscar" der Branche.

In der Gruppe der HNO-Ärzte ist 2008 die Gräser-Impf-Tablette Gra$\mathrm{zax}^{\circledR}$, wie bereits im Vorjahr, zum innovativsten Medikament gekürt worden. Die befragten Ärzte gaben als Gründe für ihre Entscheidung die gute Wirkung, die gute Anwendungsmöglichkeit in Form einer Tablette, die gute Verträglichkeit und die leichte Handhabung an.

Grazax $^{\circledR}$ ist die erste zugelassene Allergentablette, die eine spezifische Immuntherapie auch zu Hause möglich macht. Die dreijährige Behandlung kann bei einmal täglicher Anwendung eine zunehmende Toleranz des Immunsystems gegenüber Gräser- und Rog- genpollen induzieren. Das größte bisher durchgeführte Entwicklungsprogramm zur spezifischen Immuntherapie zeigte, dass die Gräser-Impf-Tablette bereits ab der ersten Therapiesaison hochsignifikant wirksam ist. Bei zweiund dreijähriger Therapie sind gesteigerte immunologische Effekte und eine weiter zunehmende Wirksamkeit nachweisbar.

Zusätzlich zum Innovationspreis für Grazax ${ }^{\circledR}$ bescheinigten die HNOFachärzte ALK Abelló, den besten Außendienst unter den Pharmaunternehmen zu haben. Diese Einschätzung beruhte vor allem auf der Kompetenz der Berater, den regelmäßigen Besuchen, dem wertvollen Informationsangebot und der freundlichen und seriösen Beratung. Weiterhin lobten die befragten Ärzte an dem Allergenspezialisten aus Wedel bei Hamburg den Kundenservice, die Produktqualität und das Unternehmensimage.

Nach Informationen von ALK-Abelló, Wedel

\section{Leitliniengerechte Ekzemtherapie}

$\mathrm{n}$ ihrer neuen Leitlinie zur Neurodermitis (www.awmf-leitlinien.de) sprechen sich die dermatologischen und allergologischen deutschen Fachgesellschaften DDG, DGAKI und GPA für den Einsatz von Calcineurininhibitoren in sensiblen Hautzonen bei Neurodermitispatienten aus. Topische Glukokortikoide können bei längerer Behandlung empfindlicher Hautareale zu Nebenwirkungen wie Hautatrophie, Striae, Purpura und perioraler Dermatitis führen. Gerade im Gesicht ist die Gefahr besonders groß. Die genannten Nebenwirkungen werden unter einer Therapie mit dem Calcineurininhibitor Pimecrolimus (Elidel ${ }^{\circledR}$ ) nicht beobachtet. Aufgrund der besonderen Galenik eignet sich Pimecrolimus insbesondere für sensible Hautareale, denn die Creme lässt sich leicht auf der Haut verteilen, zieht gut ein und glänzt nicht. Nach der Ap- plikation können Basistherapeutika, Sonnenschutzprodukte und Kosmetika appliziert werden. Pimecrolimus ist weltweit eines der am besten dokumentierten topischen Arzneimittel. Seit 1996 sind im Rahmen von klinischen Studien über 21.000 Patienten mit dem Wirkstoff behandelt worden.

„Dass die neue Leitlinie den Einsatz von Calcineurininhibitoren als FirstLine-Therapie bei sensiblen Hautzonen empfiehlt, ist eine begrüßenswerte Entscheidung. Sensible Hautzonen erfordern eine besondere, sprich eine wirksame und sichere Therapie unter Berücksichtigung einer adäquaten Galenik", kommentierte Prof. Dr. Torsten Zuberbier von der Berliner Charité die aktuelle Leitlinie.

Nach Informationen von Novartis Pharma, Nürnberg 